\section{Thrombozytenzählung nach Fonio}

H. Baum

Institut für Laboratoriumsmedizin, Mikrobiologie und Blutdepot, Regionale Kliniken Holding RKH GmbH, Ludwigsburg, Deutschland

Englischer Begriff Fonio method of counting platelets

Definition Abschätzung der Thrombozytenzahl mithilfe eines Blutausstrichs.
Beschreibung Aus der Anzahl der $\triangleright$ Thrombozyten im Verhältnis zur Anzahl der Erythrozyten im $>$ Blutausstrich kann die Thrombozytenkonzentration abgeschätzt werden. Normalerweise werden etwa 40-60 Thrombozyten auf 1000 Erythrozyten gefunden. Unter Berücksichtigung der absoluten Erythrozytenzahl kann die Thrombozytenzahl näherungsweise berechnet werden:

Thrombozytenzahl $(/ \mu \mathrm{L})=$ Thrombozyten auf 1000 Erythrozyten $\times$ Erythrozytenzahl $(/ \mu \mathrm{L}) / 1000$

Angesichts ihrer sehr großen Streubreite eignet sich diese Methode allerdings nicht für eine genaue Thrombozytenzählung und wird heute routinemäßig nicht mehr eingesetzt. 